\title{
Algorithms for Pfaffian Systems and Cohomology Intersection Numbers of Hypergeometric Integrals
}

\author{
Saiei-Jaeyeong Matsubara-Heo ${ }^{(凶)}$ and Nobuki Takayama \\ Department of Mathematics, Graduate School of Science, \\ Kobe University, Kobe, Japan \\ \{saiei, takayama\}@math.kobe-u.ac.jp
}

\begin{abstract}
In the theory of special functions, a particular kind of multidimensional integral appears frequently. It is called the Euler integral. In order to understand the topological nature of the integral, twisted de Rham cohomology theory plays an important role. We propose an algorithm of computing an invariant cohomology intersection number of a given basis of the twisted cohomology group. We also develop an algorithm of computing the Paffian system that a given basis satisfies. These algorithms are based on the fact that the Euler integral satisfies GKZ system and utilizes algorithms to find rational function solutions of differential equations. We provide software to perform this algorithm.
\end{abstract}

Keywords: Cohomology intersection numbers · GKZ hypergeometric systems · Gröbner basis

\section{Introduction}

In the study of hypergeometric functions in several variables, one often considers the integral of the following form:

$$
\langle\omega\rangle=\int_{\Gamma} h_{1}(x)^{-\gamma_{1}} \cdots h_{k}(x)^{-\gamma_{k}} x^{c} \omega,
$$

where $h_{l}(x ; z)=h_{l, z^{(l)}}(x)=\sum_{j=1}^{N_{l}} z_{j}^{(l)} x^{\mathbf{a}^{(l)}(j)}(l=1, \ldots, k)$ are Laurent polynomials in torus variables $x=\left(x_{1}, \ldots, x_{n}\right), \mathbf{a}^{(l)}(j) \in \mathbb{Z}^{n}, \gamma_{l} \in \mathbb{C}$ and $c={ }^{t}\left(c_{1}, \ldots, c_{n}\right) \in \mathbb{C}^{n}$ are parameters, $x^{c}=x_{1}^{c_{1}} \ldots x_{n}^{c_{n}}, \Gamma$ is a suitable integration cycle, and $\omega$ is an algebraic $n$-form on $V_{z}=\left\{x \in \mathbb{C}^{n} \mid x_{1} \ldots x_{n} h_{1}(x) \ldots h_{k}(x) \neq\right.$ $0\}$. As a function of the independent variable $z=\left(z_{j}^{(l)}\right)_{j, l}$, the integral (1) defines a hypergeometric function. We call the integral (1) the Euler integral.

Supported by JSPS KAKENHI Grant Number 19K14554 (the first author) and JST CREST Grant Number JP19209317 (the first and the second authors).

(C) Springer Nature Switzerland AG 2020

A. M. Bigatti et al. (Eds.): ICMS 2020, LNCS 12097, pp. 73-84, 2020.

https://doi.org/10.1007/978-3-030-52200-1_7 
We can naturally define the twisted de Rham cohomology group associated to the Euler integral (1). We set $N=N_{1}+\cdots+N_{k}, \mathbb{G}_{m}^{n}=\operatorname{Specm} \mathbb{C}\left[x_{1}^{ \pm}, \ldots, x_{n}^{ \pm}\right]$, and $\mathbb{A}^{N}=\operatorname{Specm} \mathbb{C}\left[z_{j}^{(l)}\right]$. For any $z \in \mathbb{A}^{N}$, we can define an integrable connection $\nabla_{x}=d_{x}-\sum_{l=1}^{k} \gamma_{l} \frac{d_{x} h_{l}}{h_{l}} \wedge+\sum_{i=1}^{n} c_{i} \frac{d x_{i}}{x_{i}} \wedge: \mathcal{O}_{V_{z}} \rightarrow \Omega_{V_{z}}^{1}$. The algebraic de Rham cohomology group $\mathrm{H}_{\mathrm{dR}}^{*}\left(V_{z} ;\left(\mathcal{O}_{V_{z}}, \nabla_{x}\right)\right)$ is defined as the hypercohomology group

$$
\mathrm{H}_{\mathrm{dR}}^{*}\left(V_{z} ;\left(\mathcal{O}_{V_{z}}, \nabla_{x}\right)\right)=\mathbb{H}^{*}\left(V_{z} ;\left(0 \rightarrow \mathcal{O}_{V_{z}} \stackrel{\nabla_{x}}{\rightarrow} \Omega_{V_{z}}^{1} \stackrel{\nabla_{x}}{\rightarrow} \cdots \stackrel{\nabla_{x}}{\rightarrow} \Omega_{V_{z}}^{n} \rightarrow 0\right)\right) .
$$

Under a genericity assumption on the parameters $\gamma_{l}$ and $c$, we have the vanishing result $\mathrm{H}_{\mathrm{dR}}^{m}\left(V_{z} ;\left(\mathcal{O}_{V_{z}}, \nabla_{x}\right)\right)=0(m \neq n)$. Moreover, we can define a perfect pairing $\langle\bullet, \bullet\rangle_{c h}: \mathrm{H}_{\mathrm{dR}}^{n}\left(V_{z} ;\left(\mathcal{O}_{V_{z}}, \nabla_{x}\right)\right) \times \mathrm{H}_{\mathrm{dR}}^{n}\left(V_{z} ;\left(\mathcal{O}_{V_{z}}^{\vee}, \nabla_{x}^{\vee}\right)\right) \rightarrow \mathbb{C}$ which is called the cohomology intersection form. Here, $\left(\mathcal{O}_{V_{z}}^{\vee}, \nabla_{x}^{\vee}\right)^{\vee}$ is the dual connection of $\left(\mathcal{O}_{V_{z}}, \nabla_{x}\right)$.

The study of intersection numbers of twisted cohomology groups and twisted period relations for hypergeometric functions started with the celebrated work by K. Cho and K. Matsumoto [6]. They clarified that the cohomology intersection number appears naturally as a part of the quadratic relation, a class of functional relations of hypergeometric functions. They also developed a systematic method of computing the cohomology intersection number for 1-dimensional integrals. Since this work, several methods have been proposed to evaluate intersection numbers of twisted cohomology groups, see, e.g., [2,3,10,11,14,17,19] and references therein. All methods utilize comparison theorems of twisted cohomology groups and residue calculus.

When $z$ belongs to a certain non-empty Zariski open subset of $\mathbb{A}^{N}$ (the non-singular locus), we proposed a new method in the paper [16] to obtain cohomology intersection numbers by constructing a rational function solution of a system of linear partial differential equations. One weak point of the method was that it was not algorithmic to construct the Pfaffian system (the explicit form of the integrable connection) for a given basis of the twisted cohomology group. We will give a new algorithm to construct the Pfaffian system for a given basis in this paper (Algorithm 1). To our knowledge, algorithms to find the Pfaffian system (or equation) with respect to a given basis of twisted cohomology group do not appear in the literature except the twisted logarithmic cohomology case ${ }^{1}$. Our algorithm works for a more general class of twisted cohomology groups. Moreover, it is more efficient by utilizing Saito's $b$-function [23] expressed in terms of facets of a polytope. The Sect. 2 is a brief overview of the paper [16]. The Sect. 3 is the main part and in the Sects. 4 and 5, we will give demonstrations of our implementation. As to the construction of rational function solutions, we utilize the algorithm and the implementation by M. Barkatou, T. Cluzeau, C. El Bacha, J.-A. Weil [5] (see also [4,18] and their references).

\footnotetext{
${ }^{1}$ K. Nishitani, master thesis 2011 (in Japanese), Kobe University.
} 


\section{General Results}

\subsection{The Cohomology Intersection Form}

We denote by $\mathrm{H}_{d R, c}^{n}\left(V_{z}^{a n} ;\left(\mathcal{O}_{V_{z}^{a n}}, \nabla_{x}^{a n}\right)\right)$ the analytic de Rham cohomology group with compact support. By Poincaré-Verdier duality, the bilinear pairing

$$
\begin{aligned}
& \mathrm{H}_{d R, c}^{n}\left(V_{z}^{a n} ;\left(\mathcal{O}_{V_{z}^{a n}}, \nabla_{x}^{a n}\right)\right) \times \mathrm{H}_{d R}^{n}\left(V_{z}^{a n} ;\left(\mathcal{O}_{V_{z}^{a n}}^{\vee}, \nabla_{x}^{a n \vee}\right)\right) \rightarrow \quad \mathbb{C} \\
& \Psi \quad \Psi \\
& (\phi, \psi) \quad \mapsto \int_{V_{z}^{a n}} \phi \wedge \psi
\end{aligned}
$$

is perfect. We say that the regularization condition is satisfied if the canonical morphism $\mathrm{H}_{d R, c}^{n}\left(V_{z}^{a n} ;\left(\mathcal{O}_{V_{z}^{a n}}, \nabla_{x}^{a n}\right)\right) \rightarrow \mathrm{H}_{d R}^{n}\left(V_{z}^{a n} ;\left(\mathcal{O}_{V_{z}^{a n}}, \nabla_{x}^{a n}\right)\right)$ is an isomorphism. In the following, we always assume that the regularization condition is satisfied. A criterion for this assumption is explained in Sect.2.3. Since $\left(\mathcal{O}_{V_{z}}, \nabla_{x}\right)$ is a regular connection, the canonical morphism $\mathrm{H}_{d R}^{n}\left(V_{z} ;\left(\mathcal{O}_{V_{z}}, \nabla_{x}\right)\right)$ $\rightarrow \mathrm{H}_{d R}^{n}\left(V_{z}^{a n} ;\left(\mathcal{O}_{V_{z}^{a n}}, \nabla_{x}^{a n}\right)\right)$ is always an isomorphism by Deligne-Grothendieck comparison theorem ([7, Corollaire 6.3]). Therefore, we have a canonical isomorphism reg: $\mathrm{H}_{d R}^{n}\left(V_{z} ;\left(\mathcal{O}_{V_{z}}, \nabla_{x}\right)\right) \rightarrow \mathrm{H}_{d R, c}^{n}\left(V_{z}^{a n} ;\left(\mathcal{O}_{V_{z}^{a n}}, \nabla_{x}^{a n}\right)\right)$. Note that the Poincaré dual of the isomorphism reg is called a regularization map in the theory of special functions $([2, \S 3.2])$. Finally, we define the cohomology intersection form $\langle\bullet, \bullet\rangle_{\text {ch }}$ between algebraic de Rham cohomology groups by the formula

$$
\begin{aligned}
\langle\bullet, \bullet\rangle_{c h}: \mathrm{H}_{d R}^{n}\left(V_{z} ;\left(\mathcal{O}_{V_{z}}, \nabla_{x}\right)\right) \underset{\Psi}{\times} \mathrm{H}_{d R}^{n}\left(V_{z} ;\left(\mathcal{O}_{V_{z}}^{\vee}, \nabla_{x}^{\vee}\right)\right) & \rightarrow \underset{\mathbb{C}}{(\phi, \psi)} \\
& \mapsto \int_{V_{z}^{a n}} \operatorname{reg}(\phi) \wedge \psi
\end{aligned}
$$

The value above is called the cohomology intersection number of $\phi$ and $\psi$.

\subsection{The Secondary Equation}

Now, we treat $z$ as a variable. Let $\pi: X=\left(\mathbb{G}_{m}\right)_{x}^{n} \times \mathbb{A}_{z}^{N} \backslash \bigcup_{l=1}^{k}\left\{(x, z) \mid h_{l, z^{(l)}}(x)=\right.$ $0\} \rightarrow \mathbb{A}_{z}^{N}=Y$ be the natural projection where subscripts stand for coordinates. We define an $\mathcal{O}_{Y}$-module $\mathcal{H}_{d R}^{n}$ by the hypercohomology group

$$
\mathcal{H}_{d R}^{n}=\mathbb{H}^{n}\left(X ;\left(0 \rightarrow \Omega_{X / Y}^{0} \stackrel{\nabla_{x}}{\rightarrow} \Omega_{X / Y}^{1} \stackrel{\nabla_{x}}{\rightarrow} \cdots \stackrel{\nabla_{x}}{\rightarrow} \Omega_{X / Y}^{n} \rightarrow 0\right)\right)
$$

Here, $\Omega_{X / Y}^{\bullet}$ denotes the sheaf of relative differential forms $\oplus_{|I|=\bullet} \mathcal{O}_{X} d x^{I}$ with respect to the morphism $\pi$. Since $Y$ is affine, $\mathcal{H}_{d R}^{n}$ is also identified with the sheaf $R^{n} \pi_{*}\left(\Omega_{X / Y}^{\bullet}, \nabla_{x}\right)$. For any $z \in Y$, there is a natural evaluation morphism $\mathrm{ev}_{z}: \mathcal{H}_{d R}^{n} \rightarrow \mathrm{H}_{d R}^{n}\left(V_{z} ;\left(\mathcal{O}_{V_{z}}, \nabla_{x}\right)\right)$. We define the dual object $\mathcal{H}_{d R}^{n \vee}$ by replacing $\nabla_{x}$ by $\nabla_{x}^{\vee}$ in the construction above. By the general theory of relative de Rham cohomology, there exists a non-empty Zariski open subset $U$ of $Y$ such that $\mathcal{H}_{d R}^{n} \Upsilon_{U} \simeq \mathcal{O}_{U}^{\oplus r}$. Therefore, for any global sections $\phi$ of $\mathcal{H}_{d R}^{n} \Upsilon_{U}$ and $\psi$ of $\mathcal{H}_{d R}^{n \vee} \Upsilon_{U}$, we can define the cohomology intersection number $\langle\phi, \psi\rangle_{c h}$ as a function of $z \in U$ 
by the formula $U \ni z \mapsto\left\langle\operatorname{ev}_{z}(\phi), \mathrm{ev}_{z}(\psi)\right\rangle_{c h} \in \mathbb{C}$. This actually defines a $\mathcal{O}_{U^{-}}$ bilinear map $\left.\left.\langle\bullet, \bullet\rangle_{c h}: \mathcal{H}_{d R}^{n}\right\rceil_{U} \times \mathcal{H}_{d R}^{n \vee}\right\rceil_{U} \rightarrow \mathcal{O}_{U}$.

We can equip $\mathcal{H}_{d R}^{n}$ with a structure of a $\mathcal{D}_{Y}$-module. For this purpose, we only need to define a connection $\nabla^{G M}: \mathcal{H}_{d R}^{n} \rightarrow \Omega_{Y}^{1}\left(\mathcal{H}_{d R}^{n}\right):=\Omega_{Y}^{n} \otimes \mathcal{H}_{d R}^{n}$. For any section $\phi \in \mathcal{H}_{d R}^{n}$, we define

$$
\nabla^{G M} \phi=d_{z} \phi-\sum_{j, l} \gamma_{l} \frac{x^{\mathbf{a}^{(l)}(j)}}{h_{l, z^{(l)}}(x)} d z_{j}^{(l)} \wedge \phi .
$$

Here, the superscript GM stands for "Gauß-Manin". The dual connection $\nabla^{\vee G M}: \mathcal{H}_{d R}^{n \vee} \rightarrow \Omega_{Y}^{1}\left(\mathcal{H}_{d R}^{n \vee}\right)$ is defined by replacing $\gamma_{l}$ by $-\gamma_{l}$ in (6).

The $\mathcal{D}_{Y}$-module structures of $\mathcal{H}_{d R}^{n}$ and $\mathcal{H}_{d R}^{n \vee}$ are compatible with the cohomology intersection form. Namely, for any local sections $\phi$ of $\mathcal{H}_{d R}^{n} \uparrow_{U}$ and $\psi$ of $\mathcal{H}_{d R}^{n \vee} \Upsilon_{U}$, we have

$$
d_{z}\langle\phi, \psi\rangle_{c h}=\left\langle\nabla^{G M} \phi, \psi\right\rangle_{c h}+\left\langle\phi, \nabla^{\vee G M} \psi\right\rangle_{c h}
$$

We call (7) the secondary equation. Let us rewrite it in terms of local frames. Let $\left\{\phi_{i}\right\}_{i=1}^{r}\left(\operatorname{resp} .\left\{\psi_{i}\right\}_{i=1}^{r}\right)$ be a free basis of $\mathcal{H}_{d R}^{n} \Upsilon_{U}\left(\right.$ resp. $\left.\mathcal{H}_{d R}^{n \vee} \Upsilon_{U}\right)$. We set $I=I_{c h}=\left(\left\langle\phi_{i}, \psi_{j}\right\rangle_{c h}\right)_{i, j}$ and call it the cohomology intersection matrix. On the other hand, there is a $r \times r$ matrix $\Omega$ (resp. $\Omega^{\vee}$ ) with values in 1 -forms on $U$ such that the connection $\nabla^{G M}$ (resp. $\nabla^{\vee G M}$ ) is trivialized as $d_{z}+\Omega \wedge$ (resp. $\left.d_{z}+\Omega^{\vee} \wedge\right)$. Then, the secondary equation is equivalent to the system

$$
d_{z} I={ }^{t} \Omega I+I \Omega^{\vee} .
$$

We also call (8) the secondary equation. The theorem which our algorithm is based on is the following

Theorem 1 [16]. Under the regularization condition, all the entries of the cohomology intersection matrix $I_{c h}$ are rational functions. Moreover, any rational function solution I of the secondary equation (8) is, up to a scalar multiplication, equal to $I_{c h}$.

\subsection{GKZ System Behind}

In [16], it is discussed that Theorem 1 is true for more general direct image $\mathcal{D}$-modules. However, by employing the combinatorial structure behind our integrable connection $\mathcal{H}_{d R}^{n} \Upsilon_{U}$, we can show that the cohomology intersection number in question has a rational expression with respect to $z$ and $\delta$.

Let us recall the definition of GKZ system [8]. For a given $d \times N(d<N)$ integer matrix $A=(\mathbf{a}(1)|\cdots| \mathbf{a}(N))$ and a parameter vector $\delta \in \mathbb{C}^{d}$, GKZ system $M_{A}(\delta)$ is defined as a system of partial differential equations on $\mathbb{C}^{N}$ given by

$$
M_{A}(\delta): \begin{cases}E_{i} \cdot f(z)=0 & (i=1, \ldots, d) \\ \square_{u} \cdot f(z)=0 & \left(u \in \operatorname{Ker}\left(A \times: \mathbb{Z}^{N \times 1} \rightarrow \mathbb{Z}^{d \times 1}\right)\right),\end{cases}
$$


where $E_{i}$ and $\square_{u}$ for $u={ }^{t}\left(u_{1}, \ldots, u_{N}\right)$ are differential operators defined by

$$
E_{i}=\sum_{j=1}^{N} a_{i j} z_{j} \frac{\partial}{\partial z_{j}}+\delta_{i}, \quad \square_{u}=\prod_{u_{j}>0}\left(\frac{\partial}{\partial z_{j}}\right)^{u_{j}}-\prod_{u_{j}<0}\left(\frac{\partial}{\partial z_{j}}\right)^{-u_{j}}
$$

For convenience, we assume an additional condition $\mathbb{Z} A \stackrel{\text { def }}{=} \mathbb{Z} \mathbf{a}(1)+\cdots+\mathbb{Z} \mathbf{a}(N)=$ $\mathbb{Z}^{d}$. In our setting, we put $A_{l}=\left(\mathbf{a}^{(l)}(1)|\ldots| \mathbf{a}^{(l)}\left(N_{l}\right)\right), d=n+k, N=N_{1}+\cdots+$ $N_{k}$. We define an $(n+k) \times N$ matrix $A$ by

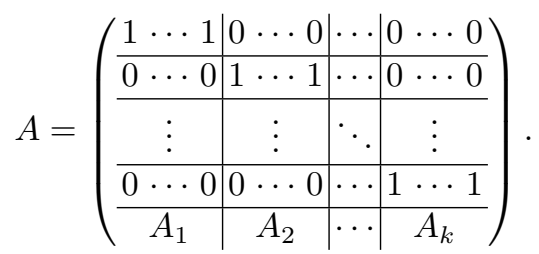

We put $\delta=\left(\begin{array}{l}\gamma \\ c\end{array}\right)$. By abuse of notation, we also denote by $M_{A}(\delta)$ the quotient $\mathcal{D}_{Y}$-module $\mathcal{D}_{Y} / J$ where $J$ is the left ideal of $\mathcal{D}_{Y}$ generated by the operators (10). It is known that GKZ system $M_{A}(\delta)$ is holonomic ([1]). We say that the parameter $\delta$ is non-resonant if it does not belong to any $\mathbb{C} \Gamma+\mathbb{Z}^{d}$ where $\Gamma$ is any facet of the cone $\sum_{j=1}^{N} \mathbb{R}_{\geq 0} \mathbf{a}(j)$. $\mathcal{H}_{d R}^{n}$ (resp. $\mathcal{H}_{d R}^{n \vee}$ ) is isomorphic to GKZ system $M_{A}(\delta)$ (resp. $M_{A}(-\delta)$ ) and the regularization condition is true when the parameter vector $\delta$ is non-resonant and $\gamma_{l} \notin \mathbb{Z}$ (see $[9,2.9]$ and $[15$, Theorem 2.12]). We set $\frac{d x}{x}=\frac{d x_{1}}{x_{1}} \wedge \cdots \wedge \frac{d x_{n}}{x_{n}}$. The isomorphism $M_{A}(\delta) \simeq \mathcal{H}_{d R}^{n}$ is given by the correspondence [1] $\mapsto\left[\frac{d x}{x}\right]$. Thus, any section $\phi$ of $\mathcal{H}_{d R}^{n}$ can be written as $\phi=P \cdot\left[\frac{d x}{x}\right]$ for some linear differential operator $P \in \mathcal{D}_{Y}$. We define the field $\mathbb{Q}(\delta)$ as the field extension $\mathbb{Q}\left(\gamma_{1}, \ldots, \gamma_{k}, c_{1}, \ldots, c_{n}\right)$ of $\mathbb{Q}$.

Theorem 2 [16]. Suppose that $A$ as in (11) admits a unimodular regular triangulation $T$ and $\delta$ is non-resonant and $\gamma_{l} \notin \mathbb{Z}$. Then, for any $P_{1}, P_{2} \in \mathbb{Q}(\delta)\left\langle z, \partial_{z}\right\rangle$, the cohomology intersection number $\frac{\left\langle P_{1} \cdot \frac{d x}{x}, P_{2} \cdot \frac{d x}{x}\right\rangle_{c h}}{(2 \pi \sqrt{-1})^{n}}$ belongs to the field $\mathbb{Q}(\delta)(z)$.

\section{An Algorithm of Finding the Pfaffian System for a Given Basis}

In this section, we set $\beta:=-\delta$. With this notation, we put $H_{A}(\beta):=M_{A}(\delta)$. This is because we use some results from [12] and [23] where the hypergeometric ideal is denoted by $H_{A}(\beta)$ while our main references $[15,16]$ denote it by $M_{A}(\delta)$.

Let $\omega_{q}$ be the differential form

$$
\prod_{l=1}^{k} h_{l}^{-q_{l}^{\prime}} x^{q^{\prime \prime}} \frac{d x}{x}, \quad q=\left(q^{\prime}, q^{\prime \prime}\right) \in \mathbb{Z}^{k} \times \mathbb{Z}^{n} .
$$

It is known that there exists a basis of the twisted cohomology group of which elements are of the form $\omega_{q}$ when $\delta$ is generic (see, e.g., [13, Th 2]). Let $\left\{\omega_{q} \mid q \in\right.$ 
$Q\}$ be a basis of the twisted cohomology group. We will give an algorithm to find the Pfaffian system $\frac{\partial}{\partial z_{i}} \omega=P_{i} \omega$ with respect to this basis $\omega=\left(\omega_{q} \mid q \in Q\right)^{T}$. Note that algorithms to translate a given holonomic ideal to a Pfaffian system are well known (see, e.g., [12, Chap 6]). However, as long as we know, algorithms to find the Pfaffian system with respect to a given basis of twisted cohomology group do not appear in the literature. Note that the pairing of the twisted homology and cohomology groups is perfect under our assumption. Then, the Pfaffian equation of the fundamental solution matrix of solutions of the GKZ system can be regarded as a relation of the twisted cycles.

Put $\partial_{i}=\frac{\partial}{\partial z_{i}}$. In this subsection, we use $\bullet$ to denote the action to avoid a confusion with the multiplication. The function $\left\langle\omega_{q}\right\rangle$ is a solution of the hypergeometric system $H_{A}(\beta-q)$. The main point of our method is of use of the following contiguity relation

$$
\frac{1}{\mathbf{a}_{i}^{\prime} \cdot(\beta-q)} \partial_{i} \bullet\left\langle\omega_{q}\right\rangle=\left\langle\omega_{q^{\prime}}\right\rangle, \quad q^{\prime}=q+\mathbf{a}_{i}
$$

where $\mathbf{a}_{i}=\mathbf{a}(\mathbf{i})$ is the $i$-th column vector of $A$ and $\mathbf{a}_{i}^{\prime}$ is the column vector that the first $k$ elements are equal to those of $\mathbf{a}_{i}$ and the last $n$ elements are 0 . For example, $\mathbf{a}_{1}^{\prime}=(1,0, \ldots, 0), \mathbf{a}_{2}^{\prime}=(1,0, \ldots, 0), \ldots, \mathbf{a}_{N_{1}+1}^{\prime}=(0,1,0, \ldots, 0)^{T}, \ldots$, $\mathbf{a}_{N}^{\prime}=(0, \ldots, 0,1)^{T}$. The relation (13) can be proved by differentiating $\left\langle\omega_{q}\right\rangle=$ $\int_{\Gamma} h_{1}^{-\gamma_{1}-q_{1}^{\prime}} \cdots h_{k}^{-\gamma_{k}-q_{k}^{\prime}} x^{c+q^{\prime \prime}} \frac{d x}{x}$, with respect to $z_{i}$ where we have $\beta-q=\left(-\gamma_{1}-\right.$ $\left.q_{1}^{\prime}, \ldots,-\gamma_{k}-q_{k}^{\prime},-c_{1}-q_{1}^{\prime \prime}, \ldots,-c_{n}-q_{n}^{\prime \prime}\right)^{T}$.

In [23, Algorithm 3.2], an algorithm to obtain an operator $C_{i}$ satisfying

$$
C_{i} \partial_{i}-b_{i}(\beta)=0 \quad \bmod H_{A}(\beta)
$$

is given. The polynomial $b_{i}$ is a $b$-function in the direction $i$ [23, Th 3.2]. Note that the algorithm outputs the operator $C_{i}$ in $\mathbb{C}\left\langle z_{1}, \ldots, z_{N}, \partial_{1}, \ldots, \partial_{N}\right\rangle$, which does not depend on the parameter $\beta$. Since $\left\langle\omega_{q}\right\rangle$ is a solution of $H_{A}(\beta-q)$, we have the following inverse contiguity relation

$$
\frac{\mathbf{a}_{i}^{\prime} \cdot\left(\beta-q^{\prime \prime}\right)}{b_{i}\left(\beta-q^{\prime \prime}\right)} C_{i} \bullet\left\langle\omega_{q}\right\rangle=\left\langle\omega_{q^{\prime \prime}}\right\rangle, \quad q^{\prime \prime}=q-\mathbf{a}_{i} .
$$

Example 1. (Gauss hypergeometric function ${ }_{2} F_{1}$.) Put

$$
A=\left(\begin{array}{ll|ll}
1 & 1 & 0 & 0 \\
\hline 0 & 0 & 1 & 1 \\
\hline 0 & 1 & 0 & 1
\end{array}\right)
$$

Then, $h_{1}=z_{1}+z_{2} x, h_{2}=z_{3}+z_{4} x$. We have

$$
\left\langle\omega_{(1,0,0)}\right\rangle=\int_{\Gamma} h_{1}^{-\gamma_{1}} h_{2}^{-\gamma_{2}} x^{c} \frac{1}{h_{1}} \frac{d x}{x} .
$$

We can show that $\left\{\omega_{(1,0,0)}, \omega_{(1,0,0)}-\omega_{(0,1,0)}\right\}$ is a basis of the twisted cohomolgy group. This $A$ is normal and the $b$-function $b_{4}(s) \in \mathbb{Q}\left[s_{1}, s_{2}, s_{3}\right]$ for the direction 
$z_{4}$ is $b_{4}(s)=s_{2} s_{3}$. Then, $C_{4}=z_{2} z_{3} \partial_{1}+\left(\theta_{2}+\theta_{3}+\theta_{4}\right) z_{4}$ where $\theta_{i}=z_{i} \partial_{i}$ by reducing $\left(\theta_{3}+\theta_{4}\right)\left(\theta_{2}+\theta_{4}\right)$ by the toric ideal $I_{A}=\left\langle\underline{\partial_{2} \partial_{3}}-\partial_{1} \partial_{4}\right\rangle$ (see Algorithm 3.2 of $[23])$.

Our algorithm to find a Pfaffian system with respect to a given basis of the twisted cohomology group is as follows.

Algorithm 1. Input: $\left\{\omega_{q} \mid q \in Q\right\}$, a basis of the twisted cohomology group. A direction (index) $i$.

Output: $P_{i}$, the coefficient matrix of the Pfaffian system $\partial_{i}-P_{i}$.

1. Compute a Gröbner basis $G$ of $H_{A}(\beta)$ in the ring of differential operators with rational function coefficients. Let $S$ be a column vector of the standard monomials with respect to $G$.

2. Put

$$
F(Q)=(F(q) \mid q \in Q)^{T}, \quad F(q)=\prod_{r_{i}<0} C_{i}^{-r_{i}} \prod_{r_{i}>0} \partial_{i}^{r_{i}} \frac{1}{B B^{\prime}}, \quad q=\sum_{i=1}^{N} r_{i} \mathbf{a}_{i}
$$

It is a vector with entries in the ring of differential operators and the order of the product is $i=N, N-1, \ldots, 3,2,1$. In other words, we apply operators from $\partial_{1}$. The polynomial $B$ is derived from the coefficient of the contiguity relation (15) and is equal to

$$
\begin{aligned}
B & =\prod_{j=1, r_{j}<0}^{N} \frac{b_{j}\left(\beta_{j}^{\prime}+\mathbf{a}_{j}\right)}{\mathbf{a}_{j}^{\prime} \cdot\left(\beta_{j}^{\prime}+\mathbf{a}_{j}\right)} \frac{b_{j}\left(\beta_{j}^{\prime}+2 \mathbf{a}_{j}\right)}{\mathbf{a}_{j}^{\prime} \cdot\left(\beta_{j}^{\prime}+2 \mathbf{a}_{j}\right)} \cdots \frac{b_{j}\left(\beta_{j}^{\prime}+\left(-r_{j}\right) \mathbf{a}_{j}\right)}{\mathbf{a}_{j}^{\prime} \cdot\left(\beta_{j}^{\prime}+\left(-r_{j}\right) \mathbf{a}_{j}\right)}, \\
\beta_{j}^{\prime} & =\beta-\sum_{r_{l}>0} r_{l} \mathbf{a}_{l}+\sum_{l=1, r_{l}<0}^{j-1}\left(-r_{l}\right) \mathbf{a}_{l} .
\end{aligned}
$$

The polynomial $B^{\prime}$ comes from the denominator of the contiguity relation (13) and is equal to

$$
\begin{aligned}
B^{\prime} & =\prod_{j=1, r_{j}>0}^{N}\left(\mathbf{a}_{j}^{\prime} \cdot\left(\beta_{j}^{\prime}\right)\right)\left(\mathbf{a}_{j}^{\prime} \cdot\left(\beta_{j}^{\prime}-\mathbf{a}_{j}\right)\right) \cdots\left(\mathbf{a}_{j}^{\prime} \cdot\left(\beta_{j}^{\prime}-\left(r_{j}-1\right) \mathbf{a}_{j}\right)\right), \\
\beta_{j}^{\prime} & =\beta-\sum_{r_{l}>0, l<j} r_{l} \mathbf{a}_{l} .
\end{aligned}
$$

3. Compute the normal form of the vectors $\partial_{i} F(Q)$ and $F(Q)$. Write the normal forms of them as $P^{\prime} S$ and $P^{\prime \prime} S$ respectively where $P^{\prime}$ and $P^{\prime \prime}$ are matrices with rational function entries.

4. Output $P_{i}=P^{\prime}\left(P^{\prime \prime}\right)^{-1}$.

The matrix $P^{\prime \prime}$ is invertible if and only if the given set of differential forms $\left\{\omega_{q}\right\}$ is a basis of the twisted cohomology group. 
We show the correctness of the algorithm. Take an element $q \in Q$. We express $\left\langle\omega_{q}\right\rangle$ in terms of $\left\langle\omega_{0}\right\rangle$, which is a solution of $H_{A}(\beta)$, by the contiguity relations (13) and (15). Note that the contiguity relations for functions $\left\langle\omega_{q}\right\rangle$ give the contiguity relations for cohomology classes $\left[\omega_{q}\right]$ by virtue of the perfectness of the pairing between the twisted homology and the twisted cohomology groups. The point of the correctness is the following identity

$$
F(q) \bullet \omega_{0}=\omega_{q}
$$

Let us illustrate how to prove (23) by examples. We assume that $q=2 \mathbf{a}_{1}+\mathbf{a}_{2}$ and $N_{1} \geq 2$. Then $\omega_{q}$ can be obtained by applying (13) with $i=1$ for two times and that with $i=2$. We have

$$
\begin{aligned}
\omega_{\mathbf{a}_{1}} & =\frac{1}{\mathbf{a}_{1}^{\prime} \cdot \beta} \partial_{1} \bullet \omega_{0}, \\
\omega_{2 \mathbf{a}_{1}} & =\frac{1}{\mathbf{a}_{1}^{\prime} \cdot\left(\beta-\mathbf{a}_{1}\right)} \partial_{1} \bullet \omega_{\mathbf{a}_{1}}, \\
\omega_{2 \mathbf{a}_{1}+\mathbf{a}_{2}} & =\frac{1}{\mathbf{a}_{2}^{\prime} \cdot\left(\beta-2 \mathbf{a}_{1}\right)} \partial_{2} \bullet \omega_{2 \mathbf{a}_{1}} .
\end{aligned}
$$

Thus, we obtain the numbers (21) and then (23). Let us consider the case that $q=-2 \mathbf{a}_{1}-\mathbf{a}_{2}$ and $N_{1} \geq 2$. Then $\omega_{q}$ can be obtained by applying (15) with $i=1$ for two times and that with $i=2$. Since $\left\langle\omega_{-\mathbf{a}_{1}}\right\rangle$ is a solution of $H_{A}\left(\beta+\mathbf{a}_{1}\right)$, we have

$$
\left[c_{1} \partial_{1}-b_{1}\left(\beta+\mathbf{a}_{1}\right)\right] \bullet \omega_{-\mathbf{a}_{1}}=0
$$

from [23]. Then, we have

$$
\begin{aligned}
\omega_{-\mathbf{a}_{1}} & =\frac{\mathbf{a}_{1}^{\prime} \cdot\left(\beta+\mathbf{a}_{1}\right)}{b_{1}\left(\beta+\mathbf{a}_{1}\right)} c_{1} \bullet \omega_{0}, \\
\omega_{-2 \mathbf{a}_{1}} & =\frac{\mathbf{a}_{1}^{\prime} \cdot\left(\beta+2 \mathbf{a}_{1}\right)}{b_{1}\left(\beta+2 \mathbf{a}_{1}\right)} c_{1} \bullet \omega_{-\mathbf{a}_{1},} \\
\omega_{-2 \mathbf{a}_{1}-\mathbf{a}_{2}} & =\frac{\mathbf{a}_{2}^{\prime} \cdot\left(\beta+2 \mathbf{a}_{1}+\mathbf{a}_{2}\right)}{b_{2}\left(\beta+2 \mathbf{a}_{1}+\mathbf{a}_{2}\right)} c_{2} \bullet \omega_{-2 \mathbf{a}_{1} .}
\end{aligned}
$$

Thus, we obtain the numbers (19) and then (23). The general case can be shown by repeating these procedures. When the normal form $F(q)$ with respect to the Gröbner basis $G$ is $\sum p_{i}^{\prime \prime} s_{i}$ where $S=\left(s_{i}\right)$ and $p_{i}^{\prime \prime}$ is a rational function in $z$ and $\beta$, we have $\omega_{q}=F(q) \bullet \omega_{0}=\sum p_{i}^{\prime \prime} s_{i} \bullet \omega_{0}$. The correctness of the last two steps follows from this fact.

Example 2. This is a continuation of Example 1. We have $(1,0,0)^{T}=\mathbf{a}_{\mathbf{1}}$ and $(0,1,0)^{T}=\mathbf{a}_{\mathbf{3}}$. Then, the basis of the twisted cohomology group $F(Q)$ is expressed as $F(Q)=\left(\partial_{1} / \beta_{1}, \partial_{1} / \beta_{1}-\partial_{3} / \beta_{2}\right)^{T}$ and $\partial_{4} F(Q)=\left(\partial_{4} \partial_{1} / \beta_{1}, \partial_{4} \partial_{1} / \beta_{1}-\right.$ $\left.\partial_{4} \partial_{3} / \beta_{2}\right)^{T}$. We can obtain a Gröbner basis whose set of the standard monomials is $\left\{\partial_{4}, 1\right\}$ by the graded reverse lexicographic order such that $\partial_{i}>$ $\partial_{i+1}$. We multiply $\beta_{1} \beta_{2}$ to $F(Q)$ and $\partial_{4} F(Q)$ in order to avoid rational polynomial arithmetic. Then, the normal form, for example, of $\beta_{2} \partial_{1}$ is 
$\frac{1}{z_{1} z_{4}-z_{2} z_{3}}\left(\left(\beta_{1}\left(\beta_{1}+\beta_{2}\right) z_{4}\right) \partial_{4}-\beta_{2}^{2} \beta_{3}\right)$. By computing the other normal forms, we obtain the matrix

$$
P_{4}=\left(\begin{array}{cc}
\frac{-\beta_{2}\left(z_{3}-z_{1}\right)}{z_{1} z_{4}-z_{2} z_{3}} & \frac{\beta_{2} z_{3}}{z_{1} z_{4}-z_{2} z_{3}} \\
\frac{-\left(\left(\beta_{2} z_{3}+\left(-\beta_{2}+\beta_{3}\right) z_{1}\right) z_{4}+\left(\beta_{1}-\beta_{3}\right) z_{2} z_{3}-\beta_{1} z_{1} z_{2}\right)}{z_{4}\left(z_{1} z_{4}-z_{2} z_{3}\right)} & \frac{\left(\beta_{2} z_{3}+\beta_{3} z_{1}\right) z_{4}+\left(\beta_{1}-\beta_{3}\right) z_{2} z_{3}}{z_{4}\left(z_{1} z_{4}-z_{2} z_{3}\right)}
\end{array}\right)
$$

\section{Implementation and Examples}

We implemented our algorithms on the computer algebra system Risa/Asir [21] with a Polymake interface. Polymake (see, e.g., [20,22]) is a system for polyhedral geometry and it is used for an efficient computation of contiguity relations ([23, Algorithm 3.2]). Here is an input ${ }^{2}$ to find the coefficient matrix $P_{4}$ for Example 1 with respect to the variable $z_{4}$ when $z_{1}=z_{2}=z_{3}=1$ (note that in our implementation $x$ is used instead of $z$ ).

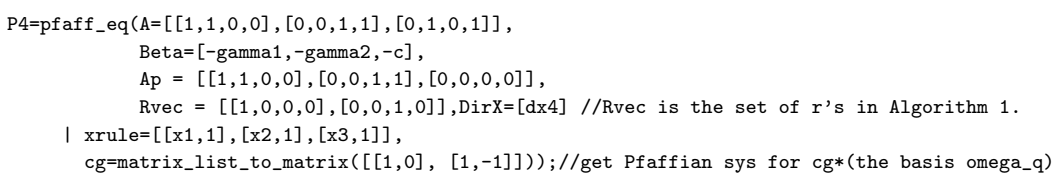

It outputs the following coefficient matrix

$$
P_{4}=\left(\begin{array}{cc}
0 & \frac{-\gamma_{2}}{x_{4}-1} \\
\frac{c}{x_{4}} & \frac{\left(-c-\gamma_{2}\right) x_{4}+c-\gamma_{1}}{\left(x_{4}-1\right) x_{4}}
\end{array}\right)
$$

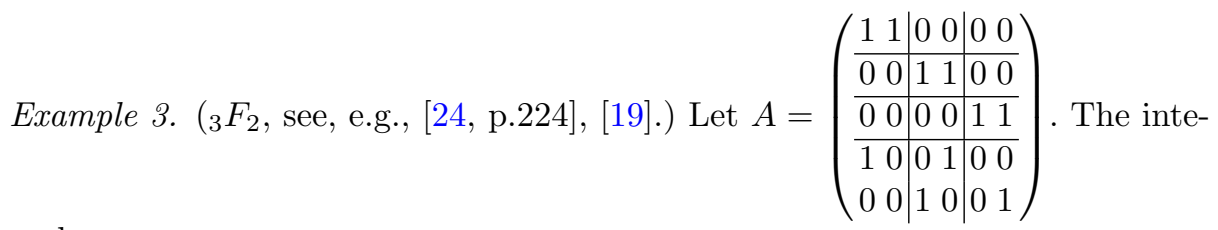
grals are

$$
\int_{\Gamma}\left(z_{1} x_{1}+z_{2}\right)^{-\gamma_{1}}\left(z_{3} x_{2}+z_{4} x_{1}\right)^{-\gamma_{2}}\left(z_{5}+z_{6} x_{2}\right)^{-\gamma_{3}} x_{1}^{c_{1}} x_{2}^{c_{2}} \omega_{i}
$$

where

$$
\omega_{1}=\frac{d x_{1} d x_{2}}{\left(z_{1} x_{1}+z_{2}\right) x_{1} x_{2}}, \omega_{2}=\frac{d x_{1} d x_{2}}{\left(z_{5}+z_{6} x_{2}\right) x_{1} x_{2}}, \omega_{3}=\frac{d x_{1} d x_{2}}{\left(z_{3} x_{2}+z_{4} x_{1}\right) x_{1} x_{2}}
$$

When $z_{2}=-1, z_{3}=z_{4}=z_{5}=z_{6}=1$, the coefficient matrix for $z_{1}$ for the basis $\left(\left\langle\omega_{1}\right\rangle,\left\langle\omega_{2}\right\rangle,\left\langle\omega_{3}\right\rangle\right)^{T}$ is

$$
P_{1}=\left(\begin{array}{ccc}
\frac{\beta_{4} z_{1}+\beta_{2}+\beta_{3}+\beta_{4}+\beta_{5}}{z_{1}\left(z_{1}-1\right)} & \frac{\beta_{3}\left(\beta_{4}-\beta_{1}-\beta_{2}\right)}{\beta_{1} z_{1}\left(z_{1}-1\right)} & \frac{\left(-\beta_{4}+1\right) \beta_{2}\left(-\beta_{2}+\beta_{4}+\beta_{5}+1\right)}{\beta_{4} \beta_{1} z_{1}\left(z_{1}-1\right)} \\
\frac{\left(\beta_{2}+\beta_{3}-\beta_{5}\right) \beta_{1}}{\beta_{3}\left(z_{1}-1\right)} & \frac{\beta_{1} z_{1}+\beta_{2}-\beta_{4}}{z_{1}\left(z_{1}-1\right)} & \frac{\left(-\beta_{4}+1\right) \beta_{2}\left(-\beta_{2}+\beta_{4}+\beta_{5}+1\right)}{\beta_{4} \beta_{3} z_{1}\left(z_{1}-1\right)} \\
\frac{\beta_{4}\left(\beta_{2}+\beta_{3}-\beta_{5}\right) \beta_{1}}{\left(-\beta_{4}+1\right) \beta_{2}\left(z_{1}-1\right)} & \frac{\beta_{4} \beta_{3}\left(\beta_{1}+\beta_{2}-\beta_{4}\right)}{\left(-\beta_{4}+1\right) \beta_{2}\left(z_{1}-1\right)} & \frac{\left(-\beta_{2}+\beta_{4}+\beta_{5}+1\right)}{z_{1}-1}
\end{array}\right)
$$

The result can be obtained in a few seconds.

\footnotetext{
${ }^{2}$ The Algorithm 1 is implemented in saito-b.rr distributed at [25].
} 


\section{An Algorithm of Finding the Cohomology Intersection Matrix}

Theorem 3 [16]. Given a matrix $A=\left(a_{i j}\right)$ as in (11) admitting a unimodular regular triangulation $T$. When parameters are non-resonant, $\gamma_{l} \notin \mathbb{Z}$ and moreover the set of series solutions by $T$ is linearly independent, the intersection matrix of the twisted cohomology group of the GKZ system associated to the matrix A can be algorithmically determined.

We denote by $\Omega_{i}$ the coefficient matrix of $\Omega$ with respect to the 1 -form $d z_{i}$. The algorithm we propose is summarized as follows.

Algorithm 2. (A modified version of the algorithm in [16]). (12).

Input: Free bases $\left\{\phi_{j}\right\}_{j} \subset \mathcal{H}_{d R}^{n} \uparrow_{U},\left\{\psi_{j}\right\}_{j} \subset \mathcal{H}_{d R}^{n \vee} \uparrow_{U}$ which are expressed as

Output: The secondary equation (8) and the cohomology intersection matrix $I_{c h}=\left(\left\langle\phi_{i}, \psi_{j}\right\rangle_{c h}\right)_{i, j}$.

1. Obtain a Pfaffian system with respect to the given bases $\left\{\phi_{j}\right\}_{j}$ and $\left\{\psi_{j}\right\}_{j}$, i.e., obtain matrices $\Omega_{i}=\left(\omega_{i j k}\right)$ and $\Omega_{i}^{\vee}=\left(\omega_{i j k}^{\vee}\right)$ so that the equalities

$$
\partial_{i} \phi_{j}=\sum_{k} \omega_{i k j} \phi_{k}, \quad \partial_{i} \psi_{j}=\sum_{k} \omega_{i k j}^{\vee} \psi_{k}
$$

hold by Algorithm 1.

2. Find a non-zero rational function solution I of the secondary equation

$$
\partial_{i} I-{ }^{t} \Omega_{i} I-I \Omega_{i}^{\vee}=0, \quad i=1, \ldots, N .
$$

To be more precise, see, e.g., [4, 5, 18] and references therein.

3. Determine the scalar multiple of I by [15, Theorem 8.1].

Example 4. This is a continuation of Example 3. We want to evaluate the cohomology intersection matrix $I_{c h}=\left(\left\langle\omega_{i}, \omega_{j}\right\rangle_{c h}\right)_{i, j=1}^{3}$. By solving the secondary equation (for example, using [5]), we can verify that $(1,1),(1,2),(2,1)$, $(2,2)$ entries of $I_{c h}$ are all independent of $z_{1}$. Therefore, we can obtain the exact values of these entries by taking a unimodular regular triangulation $T=\{23456,12456,12346\}$ and substituting $z_{1}=0$ in [15, Theorem 8.1]. Thus, we get a correct normalization of $I_{c h}$ and the matrix $\frac{I_{c h}}{(2 \pi \sqrt{-1})^{2}}$ is given by

$$
\left[\begin{array}{cc}
r_{11} & \frac{\beta_{4}+\beta_{5}}{\beta_{5} \beta_{4}\left(\beta_{2}-\beta_{4}-\beta_{5}\right)} \\
\frac{\beta_{4}+\beta_{5}}{\beta_{5} \beta_{4}\left(\beta_{2}-\beta_{4}-\beta_{5}\right)} & r_{22} \\
\frac{\beta_{4}\left(\beta_{1}+\beta_{2}-\beta_{4}-\beta_{5}\right) z_{1}-\beta_{5} \beta_{3}}{\beta_{5}\left(\beta_{4}-1\right)\left(\beta_{2}-\beta_{4}-\beta_{5}\right)\left(\beta_{2}-\beta_{4}-\beta_{5}-1\right)} & \frac{-\beta_{4} \beta_{1} z_{1}+\beta_{5}\left(\beta_{2}+\beta_{3}-\beta_{4}-\beta_{5}\right)}{\beta_{5}\left(\beta_{4}-1\right)\left(\beta_{2}-\beta_{4}-\beta_{5}\right)\left(\beta_{2}-\beta_{4}-\beta_{5}-1\right)} \\
& \frac{\beta_{4}\left(\beta_{1}+\beta_{2}-\beta_{4}-\beta_{4}\right) z_{1}-\beta_{5} \beta_{3}}{\beta_{5}\left(\beta_{4}+1\right)\left(\beta_{2}-\beta_{4}-\beta_{5}\right)\left(\beta_{2}-\beta_{4}-\beta_{5}+1\right)} \\
& \frac{-\left(\beta_{4} \beta_{1} z_{1}-\beta_{5} \beta_{2}-\beta_{5} \beta_{3}+\beta_{5} \beta_{4}+\beta_{5}\right)}{\beta_{5}\left(\beta_{4}+1\right)\left(\beta_{2}-\beta_{4}-\beta_{5}\right)\left(\beta_{2}-\beta_{4}-\beta_{5}+1\right)}
\end{array}\right.
$$


where

$$
\begin{gathered}
r_{11}=-\frac{\left(\beta_{4} \beta_{2}+\left(\beta_{4}+\beta_{5}\right) \beta_{3}\right) \beta_{1}+\beta_{4} \beta_{2}^{2}+\left(\beta_{4} \beta_{3}-\beta_{4}^{2}-\beta_{5} \beta_{4}\right) \beta_{2}+\left(-\beta_{4}^{2}-\beta_{5} \beta_{4}\right) \beta_{3}}{\beta_{5} \beta_{4} \beta_{1}\left(\beta_{2}-\beta_{4}-\beta_{5}\right)\left(\beta_{2}+\beta_{3}-\beta_{5}\right)} \\
r_{22}=-\frac{\left(\beta_{5} \beta_{2}+\left(\beta_{4}+\beta_{5}\right) \beta_{3}-\beta_{5} \beta_{4}-\beta_{5}^{2}\right) \beta_{1}+\beta_{5} \beta_{2}^{2}+\left(\beta_{5} \beta_{3}-\beta_{5} \beta_{4}-\beta_{5}^{2}\right) \beta_{2}}{\beta_{5} \beta_{4} \beta_{3}\left(\beta_{2}-\beta_{4}-\beta_{5}\right)\left(\beta_{1}+\beta_{2}-\beta_{4}\right)} \\
r_{33}=-\frac{\beta_{4}\left\{a_{0} z_{1}{ }^{2}-2 \beta_{1} \beta_{3} \beta_{4} \beta_{5} z_{1}+a_{2}\right\}}{\beta_{5} \beta_{2}\left(\beta_{4}-1\right)\left(\beta_{4}+1\right)\left(\beta_{2}-\beta_{4}-\beta_{5}\right)\left(\beta_{2}-\beta_{4}-\beta_{5}-1\right)\left(\beta_{2}-\beta_{4}-\beta_{5}+1\right)} \\
a_{0}=\left(\beta_{1} \beta_{2}-\beta_{1} \beta_{5}+\beta_{2}{ }^{2}-\beta_{2} \beta_{4}-2 \beta_{2} \beta_{5}+\beta_{4} \beta_{5}+\beta_{5}{ }^{2}\right) \beta_{1} \beta_{4} \\
a_{2}=\left(\beta_{2}{ }^{2}+\beta_{2} \beta_{3}-2 \beta_{2} \beta_{4}-\beta_{2} \beta_{5}-\beta_{3} \beta_{4}+\beta_{4}{ }^{2}+\beta_{4} \beta_{5}\right) \beta_{3} \beta_{5}
\end{gathered}
$$

Example 5. Let $A=\left(\begin{array}{lllll}1 & 1 & 1 & 1 & 1 \\ 0 & 1 & 0 & 2 & 0 \\ 0 & 0 & 1 & 0 & 2\end{array}\right)$. The integrals are

$$
\int_{\Gamma} h_{1}^{-\gamma_{1}} x_{1}^{c_{1}} x_{2}^{c_{2}} \omega_{i}, \quad h_{1}=z_{1}+z_{2} x_{1}+z_{3} x_{2}+x_{4} x_{1}^{2}+z_{5} x_{2}^{2}
$$

where

$$
\omega_{1}=\frac{d x_{1} d x_{2}}{x_{1} x_{2}}, \omega_{2}=x_{1} \omega_{1}=\frac{d x_{1} d x_{2}}{x_{2}}, \omega_{3}=x_{2}^{2} \omega_{1}=\frac{x_{2} d x_{1} d x_{2}}{x_{1}}, \omega_{4}=x_{1} x_{2} \omega_{1}=d x_{1} d x_{2} .
$$

Note that this $A$ is not normal. When $z_{1}=z_{4}=z_{5}=1$, we have obtained the coefficient matrices $P_{2}$ and $P_{3}$ in about $9 \mathrm{~h} 45 \mathrm{~min}$ on a machine with $\operatorname{Intel}(\mathrm{R})$ Xeon(R) CPU E5-4650 2.70 GHz and 256 GB memory. The $(1,1)$ element of $P_{2}$ is

$$
\frac{\left(\left(b_{2} z_{2}^{2}+b_{123}\right) z_{3}^{2}+b_{2} z_{2}^{4}+b_{132} z_{2}^{2}-32 b_{1}+16 b_{2}+16 b_{3}-16\right)}{z_{2}\left(z_{2}-2\right)\left(z_{2}+2\right)\left(z_{3}^{2}+z_{2}^{2}-4\right)}
$$

where $b_{1}=-\gamma_{1}, b_{2}=-c_{1}, b_{3}=-c_{2}$ and $b_{i j k}=8 b_{i}-4 b_{j}-8 b_{k}+4$. A complete data of $P_{2}$ and $P_{3}$ is at [25]. The intersection matrix can be obtained by [5] in a few seconds when we specialize $b_{i}$ 's to rational numbers. See [25] as to Maple inputs for it.

\section{References}

1. Adolphson, A.: Hypergeometric functions and rings generated by monomials. Duke Math. J. 73, 269-290 (1994)

2. Aomoto, K., Kita, M.: Theory of Hypergeometric Functions. Springer, Tokyo (1994). https://doi.org/10.1007/978-4-431-53938-4. (in Japanese)

3. Aomoto, K., Kita, M.: Theory of Hypergeometric Functions. Springer, Tokyo (2011). (English translation of [2])

4. Barkatou, M.: On rational solutions of systems of linear differential equations. J. Symb. Comput. 28, 547-567 (1999) 
5. Barkatou, M., Cluzeau, T., El Bacha, C., Weil, J.-A.: IntegrableConnections - a maple package for computing closed form solutions of integrable connections (2012). https://www.unilim.fr/pages_perso/thomas.cluzeau/Packages/ IntegrableConnections/PDS.html

6. Cho, K., Matsumoto, K.: Intersection theory for twisted cohomologies and twisted Riemann's period relations I. Nagoya Math. J. 139, 67-86 (1995)

7. Deligne, P.: Equations Différentielles à Points Singuliers Réguliers. LNM, vol. 163. Springer, Heidelberg (1970). https://doi.org/10.1007/BFb0061194

8. Gel'fand, I.M., Kapranov, M.M., Zelevinsky, A.V.: Hypergeometric functions and toric varieties. Funktsional. Anal. i Prilozhen. 23(2), 12-26 (1989). Translation in Functional Analysis and Applications 23(2), 94-106 (1989)

9. Gel'fand, I.M., Kapranov, M.M., Zelevinsky, A.V.: Generalized Euler integrals and A-hypergeometric functions. Adv. Math. 84, 255-271 (1990)

10. Goto, Y., Kaneko, J., Matsumoto, K.: Pfaffian of Appell's hypergeometric system $F_{4}$ in terms of the intersection form of twisted cohomology groups. Publ. Res. Inst. Math. Sci. 52, 223-247 (2016)

11. Goto, Y., Matsumoto, K.: Pfaffian equations and contiguity relations of the hypergeometric function of type $(k+1, k+n+2)$ and their applications. Funkcialaj Ekvacioj 61, 315-347 (2018)

12. Hibi, T. (ed.): Gröbner Bases. Statistics and Software Systems. Springer, Tokyo (2013). https://doi.org/10.1007/978-4-431-54574-3. https://www.springer.com/gp/book/9784431545736

13. Hibi, T., Nishiyama, K., Takayama, N.: Pfaffian systems of $A$-hypergeometric equations I: bases of twisted cohomology groups. Adv. Math. 306, 303-327 (2017)

14. Kita, M., Yoshida, M.: Intersection theory for twisted cycles. Mathematische Nachrichten 166, 287-304 (1994)

15. Matsubara-Heo, S.-J.: Euler and Laplace integral representations of GKZ hypergeometric functions (2019). arXiv:1904.00565

16. Matsubara-Heo, S.-J., Takayama, N.: An algorithm of computing cohomology intersection number of hypergeometric integrals (2019). arXiv:1904.01253

17. Matsumoto, K.: Intersection numbers for logarithmic $k$-forms. Osaka J. Math. 35, 873-893 (1998)

18. Oaku, T., Takayama, N., Tsai, H.: Polynomial and rational solutions of holonomic systems. J. Pure Appl. Algebra 164, 199-220 (2001)

19. Ohara, K., Sugiki, Y., Takayama, N.: Quadratic relations for generalized hypergeometric functions ${ }_{p} F_{p-1}$. Funkcialaj Ekvacioj 46, 213-251 (2003)

20. Polymake - polymake is open source software for research in polyhedral geometry. https://polymake.org

21. Risa/Asir, a computer algebra system. http://www.math.kobe-u.ac.jp/Asir, http://www.openxm.org

22. Assarf, B.: Computing convex hulls and counting integer points with polymake. Math. Program. Comput. 9, 1-38 (2017)

23. Saito, M., Sturmfels, B., Takayama, N.: Hypergeometric polynomials and integer programming. Compositio Mathematica 115, 185-204 (1999)

24. Saito, M., Sturmfels, B., Takayama, N.: Gröbner Deformations of Hypergeometric Differential Equations. Springer, Heidelberg (2000). https://doi.org/10.1007/9783-662-04112-3

25. Rational function solutions and intersection numbers (software appendix of this paper). http://www.math.kobe-u.ac.jp/OpenXM/Math/intersection2 